\title{
Conventional routine clinical review may not be necessary after uncomplicated phacoemulsification
}

Bruce D S Allan, Roger M Baer, Peter Heyworth, I Graham M Duguid, John K G Dart

\begin{abstract}
Aim-To determine the clinical intervention rate during routine review after uncomplicated phacoemulsification.

Methods-A review of case notes in 651 consecutive cases of uncomplicated phacoemulsification from $1994(\leqslant 5.5 \mathrm{~mm}$ self sealing wound) was performed. The intervention rate at scheduled routine review visits and at unscheduled visits to the eye casualty service in the first 120 postoperative days was recorded. Interventions were defined as departures from predetermined postoperative care protocols.

Results-Clinical interventions were reported in $2.8 \%$ (95\% confidence interval 1.5 to $4.1 \%)$ of $(n=1652)$ routine follow up visits. Many of these interventions were avoidable or trivial; $90 \%$ of patients had no postoperative intervention at any visit. $7.3 \%$ of patients made unscheduled visits to the emergency service. The intervention rate in this group was $50 \%$ (35.9 to $64.1 \%$ ).

Conclusions-The intervention rate in routine clinical review after uncomplicated modern cataract surgery is low. Alternatives to conventional postoperative review, including shared care with nonophthalmologists and improved perioperative patient education with an open channel for self referral, should be evaluated.

(Br F Ophthalmol 1997;81:548-550)
\end{abstract}

Phacoemulsification techniques and foldable intraocular lens implants allow cataract surgery to be performed safely through a small $(3.2-5.5 \mathrm{~mm})$ self sealing pocket incision. No sutures are required. The bursting strength of $5.5 \mathrm{~mm}$ scleral pocket wounds exceeds that of the globe itself at its weakest point. ${ }^{1}$ Wound integrity can be assessed definitively at the time of surgery. Subsequent wound related complications are rarely seen, and restrictions on postoperative activity are minimal. ${ }^{2}$

Phacoemulsification is quickly becoming the preferred technique of cataract extraction, ${ }^{3}$ but routine postoperative follow up protocols in many departments are still largely based on the need for suture management and ongoing assessment of wound integrity associated with conventional extracapsular cataract extraction through a sutured $10-12 \mathrm{~mm}$ wound. As with other surgical procedures, ${ }^{4}$ follow up practices vary widely ${ }^{5}$ and preferred practice recommendations ${ }^{6}$ are not evidence based.

Cataract extraction is the most commonly performed elective operation, ${ }^{7}$ and any reduction in the requirement for routine review may have a significant health economic impact. The value of routine postoperative review is defined, at least in part, by the number and nature of clinical interventions that are occasioned at each visit. We set out to examine the intervention rate in routine follow up after uncomplicated phacoemulsification.

\section{Methods}

STUDY DESIGN

A retrospective review of case notes was performed in which the number and nature of clinical interventions occurring during routine follow up visits were recorded. An intervention was defined as any departure from predetermined routine postoperative care protocols. Data were retrieved from a 120 day postoperative follow up period, this being the maximum period for which routine review would normally have been continued. Interventions resulting from unscheduled visits to the eye casualty service between routine appointments were also recorded along with details of patient age and sex, coexisting pathology (in addition to cataract), the operating surgeon, operative complications, and the final visual outcome.

\section{STUDY POPULATION}

Consecutive cases of cataract extraction by phacoemulsification through a $4.0-5.5 \mathrm{~mm}$ self sealing corneal or scleral pocket incision performed at Moorfields Eye Hospital in 1994 were identified from theatre records. Operations were performed by consultants or surgeons in training. Cases with pre-existing ocular pathology in addition to cataract or any intraoperative complication were excluded. In patients with two eyes operated on within the study period, only the first eye was included for study.

SURGICAL TECHNIQUE AND ROUTINE FOLLOW UP PROTOCOL

Cases were performed under local (peribulbar or topical) or general anaesthesia using standard endolenticular phacoemulsification techniques. Wound positioning was determined by lens type and surgeon preference (Fig 1). At the end of surgery, approximate physiological intraocular pressure was restored with balanced salt solution injection through a side
Accepted for publication 26 March 1997 


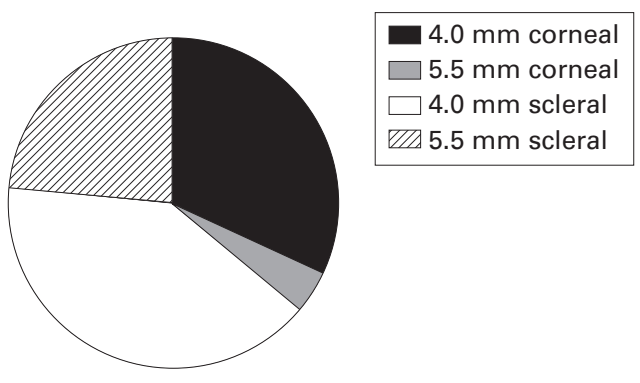

Figure 1 Relative proportions of different self sealing wound types used. Wound construction was determined by lens type and surgical preference. Rigid all polymethylmethacrylate $5 \times 6 \mathrm{~mm}(5.5 \mathrm{~mm}$ wounds $-M Z$ $60 \mathrm{BD}$, Iolab, UK) and foldable silicone $(4 \mathrm{~mm}$ wounds - L 141U, Iolab, UK) lenses were used.

port paracentesis incision. Subconjunctival cefuroxime $(125 \mathrm{mg} / \mathrm{ml})$ and betamethasone $(4 \mathrm{mg} / \mathrm{ml})$ injections were then administered. Ocular hypotensive prophylaxis (for example, acetazolamide $250 \mathrm{mg}$ orally 4 hours after surgery) was prescribed routinely by some surgeons only ( $27 \%$ of cases).

The follow up protocol was modified during the study period, reducing the number of routine review visits from three to two. Day cases and inpatients were initially reviewed on day 1 , 2 weeks, and 3 months after surgery. Later in the study period day cases were reviewed on the day of surgery ${ }^{8}$ and discharged after a single additional review visit 3 weeks after surgery. Inpatients were then also reviewed on two occasions only-on day 1 and 3 weeks after surgery.

Postoperative medication was routinely prescribed as follows: chloramphenicol eyedrops $(0.5 \%)$ and dexamethasone eyedrops $(0.1 \%)$ four times daily until the second review (2 to 3 weeks after surgery), then dexamethasone

Table 1 Clinical interventions at routine review

\begin{tabular}{llr}
\hline 1st review: & $(100 \%$ of patients; day 0-17\%; day 1-83\%) & 15 \\
Raised IOP & Ocular hypotensive medication & 6 \\
Uveitis & Increased topical steroid medication & \\
2nd review: & (100\% of patients; average 17 (SD 6.1) days postop) & 5 \\
Raised IOP & Ocular hypotensive medication & 2 \\
Uveitis & Increased topical steroid medication & 5 \\
Incidental pathology & Referral to another specialty service & 1 \\
Blepharitis & Lid hygiene instruction & 1 \\
CMO & Orbital floor steroid injection & \\
3rd review: & (48\% of patients; average 53 (SD 20.7) days postop) & \\
Uveitis & Topical steroid medication restarted & 4 \\
Capsular opacification & YAG capsulotomy & 1 \\
Lens material in AC & Lens material removed in theatre & 4 \\
Incidental pathology & Referral to another specialty service & 46 \\
Total interventions in 1652 visits & &
\end{tabular}

Clinical interventions were defined as any departure from predetermined routine postoperative management protocols. Uveitis = intraocular inflammation greater than expected for the stage in the postoperative course. $\mathrm{CMO}=$ cystoid macular oedema. $\mathrm{AC}=$ anterior chamber. Incidental pathology refers to the diagnosis of ocular pathology unrelated to cataract surgery.

Table 2 Clinical interventions in the eye casualty service

\begin{tabular}{llr}
\hline Uveitis & Topical steroid medication increased & 13 \\
Blepharitis & Lid hygiene instruction/tetracycline & 5 \\
Conjunctivitis & Antibiotic medication restarted & 2 \\
Retinal detachment & Referral to vitreoretinal service & 2 \\
Wound site inflammation & Topical steroid medication restarted & 1 \\
Incidental pathology & Referral to specialty service & 1 \\
Total interventions in 48 visits & & 24 \\
\hline
\end{tabular}

Half of the patients (total $=48$ ) presenting to the casualty service required advice and reassurance only after examination and no active therapeutic or investigational intervention. Definitions for interventions in the remaining cases are as for Table 1. alone reducing by one drop per day per week over the succeeding 4 weeks.

\section{Results}

A record of at least two routine follow up visits was available in over $96 \%$ of the total number $(\mathrm{n}=815)$ of cases identified from theatre records. After exclusions, 651 uncomplicated cases of phacoemulsification (mean age 68.5 years, range 19-99, SD = 14, female:male 1.3) were studied further. Almost all cases were age-related cataracts including a full spectrum of different nucleus densities. The population characteristics (age and sex ratio) were similar among the study sample and patients excluded from the study.

Clinical interventions were reported in $2.8 \%$ (95\% confidence limits 1.5 to $4.1 \%$ ) of $(n=1652)$ routine follow up visits (Table 1); $7.3 \%$ of patients $(n=48)$ made unscheduled visits to the emergency service. The intervention rate in this group was $50 \%$ (35.9 to $64.1 \%)$ (Table 2$) ; 93.5 \%$ of patients $(n=609)$ had no intervention during routine follow up, and $90 \%$ of patients $(n=587)$ had no postoperative intervention in either the emergency service or at scheduled review visits. Three cases $(\leqslant 0.5 \%)$ had a best corrected visual acuity less than $6 / 12$ at the last review. There were no wound related complications and no cases of endophthalmitis.

\section{Discussion}

The timing of the development of serious postoperative complications after cataract surgery cannot be predicted with any accuracy. Retinal detachment may present over a year after surgery. ${ }^{910}$ Similarly, endophthalmitis may occur late in the postoperative period, with a significant proportion of cases presenting outside the first postoperative week. ${ }^{11}{ }^{12}$ It is, therefore, virtually impossible to plan an economically viable schedule for postoperative review designed to detect serious complications at a presymptomatic stage. Many of the patients whose treatment was modified at visits 2 and 3 had already been symptomatic for a variable period. Patients may actually wait for a scheduled routine review visit rather than presenting early with a significant symptomatic problem. In contrast with the low rate of clinical intervention at routine review, the intervention rate in patients presenting to the eye casualty service between scheduled visits was $50 \%$. A more realistic approach may be to base routine follow up protocols on the detection of treatable, asymptomatic complications, with an open channel for immediate self referral where symptomatic problems develop.

The only common silent complication of uncomplicated sutureless phacoemulsification is raised intraocular pressure. Untreated pressure elevations may cause irreversible optic nerve damage. ${ }^{13-15}$ Intraocular pressure may rise temporarily in the immediate postoperative period, ${ }^{14}$ or later as a side effect of topical steroid medication. ${ }^{13}$ Prophylactic short term ocular hypotensive medication has been shown to be effective in guarding against early pressure spikes, ${ }^{16}{ }^{17}$ and in our study it may 
have been possible to avoid up to one third $(n=15)$ of all the interventions recorded in routine review if ocular hypotensive prophylaxis had been prescribed in all cases. It may also be possible to circumvent the problem of steroid induced intraocular pressure rises. Recent evidence suggests that topical nonsteroidal anti-inflammatory drugs may be as effective as steroids in suppressing inflammation after cataract surgery. ${ }^{18}{ }^{19}$ Where topical steroids are used, early refractive stability after sutureless phacoemulsification ${ }^{20}{ }^{21}$ should allow screening for a steroid induced pressure rise to be combined with postoperative refraction, at 2-3 weeks after surgery. Whether or not this combined refraction and intraoperative pressure examination would need to be performed by an ophthalmologist is a matter for debate. 2223

Moorfields Eye Hospital is a postgraduate teaching hospital with a large primary referral base from which the vast majority of patients included for study were drawn. In the context of an intervention rate study in routine clinical follow up, the retrospective design used here may well provide a better reflection of reality in clinical practice than prospective studies in which decisions about intervention may be influenced by investigator bias. A very high rate of data retrieval was achieved. Errors of omission or variable definition should have been minimal since the main outcome measure (intervention rate) simply requires that some record was made in the notes (not necessarily an accurate one) of any therapeutic intervention. A similarly low intervention rate $(3 \%)$ has recently been reported in a smaller scale prospective study examining the value of routine review on day 1 after phacoemulsification. $^{24}$

This study focuses on uncomplicated sutureless phacoemulsification, and does not address other techniques of cataract extraction or the review requirements for complicated cases. Within these confines, the striking finding was the number of review visits $(\geqslant 95 \%)$ at which nothing was achieved beyond perhaps mutual reassurance. Clearly, reassurance is important, but prioritisation is becoming increasingly necessary in many healthcare settings, and screening patients with a low yield of complications may become difficult to justify, ${ }^{25}$ particularly where time could be freed for decision making in more complex cases. Alternatives to conventional postoperative review, including shared care with nonophthalmologists and improved perioperative patient education with an open channel for self referral, should be evaluated.
We would like to acknowledge the help of Mr S J Tuft and Mr A $\mathrm{D}$ McG Steele who allowed the study group to include patients operated on under their care.

1 Ernest PH, Kiessling LA, Lavery KT. Relative strength of cataract incisions in cadaver eyes. F Cataract Refract Surg 1991;17 (Suppl):668-71.

2 Perkins RS, Olson RS. A new look at postoperative instructions following cataract extraction. Ophthalmic Surg 1991; 22:66-8.

3 Leaming DV. Practice styles and preferences of ASCRS members-1994 survey. F Cataract Refract Surg 1995;21: 378-85.

4 Emberton M. Outpatient follow up. Who benefits: doctors or patients? BMF 1995;311:1315-6.

5 Desai P. The national cataract surgery survey 3. Process features. Eye 1993;7:667-71.

6 Royal College of Ophthalmologists. Guidelines for cataract surgery. London: RCO, 1995.

7 Steinberg EP, Javitt JC, Sharkey PD, Zuckerman A, Legro MW, Anderson GF, et al. The content and cost of cataract surgery. Arch Ophthalmol 1993;111:1041-9.

8 Tufail A, Foss AJE, Hamilton AMP. Is the first day postoperative review necessary after cataract extraction? $\mathrm{Br} \mathcal{F}$ Ophthalmol 1995;79:646-8.

9 Nielsen NE, Naeser K. Epidemiology of retinal detachment following extracapsular cataract extraction. A follow up study with an analysis of risk factors. $\mathcal{F}$ Cataract Refract Surg 1993;19:675-80.

10 Coonan P, Fung WE, Webster RG, Allen AW, Abbott RL. The incidence of retinal detachment following extracapsular cataract surgery:a ten year study. Ophthalmology 1985;92:1096-101.

11 Hughes DS, Hill RJ. Infectious endophthalmitis after cataract surgery. Br F Ophthalmol 1994;78:227-32.

12 Fish A, Salvanet A, Prazuck T, Forestier F, Gerband K, Coscas G. Epidemiology of infective endophthalmitis in France. Lancet 1991;338:1373-6.

13 Hayreh SS. Anterior ishaemic optic neuropathy. IV Occurrence after cataract extraction. Arch Ophthalmol 1980;98:1410-6.

14 Rich WJ, Radke ND, Cohan BE. Early ocular hypertension after cataract extraction. Br f Ophthalmol 1974;58:725-31.

15 Kass MA, Johnson T. Corticosteroid induced glaucoma. In: Ritch R, Shields MB, Krupin T, eds. The glaucomas. St Louis: CV Mosby, 1989:1161-8.

16 Wiles SB, MacKenzie D, Ide CH. Control of intraocular pressure with apraclonidine hydrochloride after cataract extraction. Am f Ophthalmol 1989;111:184-8.

17 Feist RM, Palmer DJ, Fiscella R, Ernest JT, Tripathi R, Torczynski E, et al. Effectiveness of apraclonidine and acetazolamide in preventing postoperative intraocular pressure spikes after extracapsular cataract extraction. $f$ Cataract Refract Surg 1995;21:191-5.

18 Othenin-Girard P, Tritten JJ, Pittet N, Herbert CP. Dexamethasone versus diclofenac sodium eyedrops to treat inflammation after cataract surgery. $\mathcal{F}$ Cataract Refract Surg 1994;20:9-12.

19 Flach AJ, Kraff MC, Sanders DR, Tanenbaum L. The quantitative effect of $0.5 \%$ keratolac trimethamine solution and $0.1 \%$ dexamethasone sodium phosphate solution in postsurgical blood aqueous barrier. Arch Ophthalmol 1988; 106:480-3.

20 Kondrot EC. Keratometric cylinder and visual recovery following phacoemulsification and intraocular lens implantation using a self-sealing cataract incision. $\mathcal{F}$ Cataract Refract Surg 1991;17 (suppl):731-3.

21 Feil SH, Crandall AS, Olson RJ. Astigmatic decay following small incision self sealing cataract surgery: one year followup. I Cataract Refract Surg 1995;21:433-6.

22 Lichter P. Different providers and different error rates in health care outcomes:cataract co-manangement at what price? Ophthalmology 1993;100:445-6.

23 Revicki DA, Brown RE, Adler MA. Patient outcomes with co-managed postoperative care after cataract surgery. 7 Clin Epidemiol 1993;46:5-15.

24 Whitefield L, Crowston J, Little BC. First day follow up for routine phacoemulsification? $\mathrm{Br} \mathcal{F}$ Ophthalmol 1996;86: $148-50$.

25 Cairns J. The costs of prevention. BMf 1995;311:1520. 